\title{
Generalization of Levi-Civita regularization in the restricted three-body problem
}

\author{
R. Roman ${ }^{1}$ and I. Szücs-Csillik ${ }^{1}$ \\ Astronomical Institute of Romanian Academy, Astronomical Observatory Cluj-Napoca, \\ Str. Ciresilor No. 19, RO-400487 Cluj-Napoca, Romania \\ rdcroman@yahoo.com \\ iharka@gmail.com
}

Received — $—$ accepted $—$

Not to appear in Nonlearned J., 45. 


\begin{abstract}
A family of polynomial coupled function of $n$ degree is proposed, in order to generalize the Levi-Civita regularization method, in the restricted three-body problem. Analytical relationship between polar radii in the physical plane and in the regularized plane are established; similar for polar angles. As a numerical application, trajectories of the test particle using polynomial functions of $2,3, \ldots, 8$ degree are obtained. For the polynomial of second degree, the Levi-Civita regularization method is found.
\end{abstract}

Subject headings: celestial mechanics: regularization: restricted three-body problem 


\section{Introduction}

The regularization (in Celestial Mechanics) is a transformation of space and time variables, in order to eliminate the singularities occurring in equations of motion. As Szebehely show (see Szebehely (1967)), the purpose of regularization is to obtain regular differential equations of motion and not regular solutions.

The regularization was introduced by Levi-Civita in 1906 (see Levi-Civita (1906)) in plane, and generalized by Kustaanheimo and Stiefel in 1965 (see Kustaanheimo and Stiefel (1965)) in space. At the beginning, the regularization was developed for studying the singularities of Kepler motion, for analyzing the collisions of two point masses, and for improving the numerical integration of near-collision orbits. Many studies of the regularization problem are in the restricted three-body problem, where there are two singularities. We can regularize local (one of them), or global. Birkhoff (1915), Thiele (1896), Burrau (1906), Lemaître (1955), Arenstorf (1963), Érdi (2004), Szücs-Csillik and Roman (2012), and many other researchers studied the regularization of the restricted three-body problem.

In order to obtain the regularized equations of motion, one introduces a generating

function $S$, which depends on two harmonic and conjugated functions $f$ and $g$. But there are many harmonic and conjugated functions. Using different couples of polynomial functions, one can obtain different methods of regularization. For the polynomial of second degree we obtain the Levi-Civita regularization method. By consequence, in this article we created a class of regularization methods, which all have in common the idea that $f$ and $g$ are harmonic and conjugate polynomial functions. We studied analytically some properties of these methods.

Starting from the graphical representation of the test particle's trajectory in the circular restricted three-body problem in the physical plane, and imposing a set of initial 
conditions, we obtained trajectories in the regularized plane, using 7 regularization methods. So, the methods can be compared not only by canonical equations of motion, but also by the shape of the obtained trajectories.

\section{The restricted three-body problem}

First of all, let us analyze why the regularization is useful in the restricted three-body problem. For simplicity, we shall consider in the following that the third body moves into the orbital plane $(z=0)$. Denoting $S_{1}$ and $S_{2}$ the components of the binary system (whose masses are $m_{1}$ and $m_{2}$ ), the equations of motion of the test particle (in the frame of the restricted three-body problem) in the coordinate system $\left(x, S_{1}, y\right)$, (the physical plane) are (see equations (1)-(2) in Roman et al. (2012), Roman (2011)):

$$
\begin{gathered}
\frac{d^{2} x}{d t^{2}}-2 \frac{d y}{d t}=x-\frac{q}{1+q}-\frac{x}{(1+q) r_{1}^{3}}-\frac{q(x-1)}{(1+q) r_{2}^{3}} \\
\frac{d^{2} y}{d t^{2}}+2 \frac{d x}{d t}=y-\frac{y}{(1+q) r_{1}^{3}}-\frac{q y}{(1+q) r_{2}^{3}}
\end{gathered}
$$

where

$$
r_{1}=\sqrt{x^{2}+y^{2}}, \quad r_{2}=\sqrt{(x-1)^{2}+y^{2}}, \quad q=\frac{m_{2}}{m_{1}} .
$$

These equations have singularities in terms $\frac{1}{r_{1}}$ and $\frac{1}{r_{2}}$ (see Csillik 2003), Mioc and Csillik (2002)). This situation corresponds to collision of the test particle with $S_{1}$ and $S_{2}$. If the test particle approaches very closely to one of the primaries, such an event produces large gravitational force and sharp bends of orbit. Removing of these singularities can be done by regularization.

In order to regularize equations (1)-(2), we introduce the generalized coordinates $q_{1}$, $q_{2}$, and the generalized momenta $p_{1}, p_{2}$ (see Boccaletti and Pucacco (1996) p. 266; Roman 
et al. (2012) ), and write the Hamiltonian and canonical equations of motion:

$$
\mathcal{H}=\frac{1}{2}\left(p_{1}^{2}+p_{2}^{2}\right)+p_{1} q_{2}-q_{1} p_{2}+\frac{q_{1}^{2}}{2}+\frac{q_{2}^{2}}{2}-\psi\left(q_{1}, q_{2}\right) .
$$

where

$$
\psi\left(q_{1}, q_{2}\right)=\frac{1}{2}\left[\left(q_{1}-\frac{q}{1+q}\right)^{2}+q_{2}^{2}+\frac{2}{(1+q) r_{1}}+\frac{2 q}{(1+q) r_{2}}\right]
$$

with

$$
r_{1}=\sqrt{q_{1}^{2}+q_{2}^{2}}, \quad r_{2}=\sqrt{\left(q_{1}-1\right)^{2}+q_{2}^{2}} .
$$

Here the generalized coordinates and the generalized momenta were:

$$
q_{1}=x, \quad q_{2}=y, \quad p_{1}=\dot{q}_{1}-q_{2}, \quad p_{2}=\dot{q}_{2}+q_{1} .
$$

The canonical equations have the general form:

$$
\dot{q}_{i}=\frac{\partial \mathcal{H}}{\partial p_{i}}, \quad \dot{p}_{i}=-\frac{\partial \mathcal{H}}{\partial q_{i}}, \quad i \in\{1,2\} .
$$

The canonical equations obtained from equations (1)-(2) have, in the $\left(q_{1}, S_{1}, q_{2}\right)$ coordinate system, the explicit form:

$$
\begin{aligned}
\frac{d q_{1}}{d t} & =p_{1}+q_{2} \\
\frac{d q_{2}}{d t} & =p_{2}-q_{1} \\
\frac{d p_{1}}{d t} & =p_{2}-\frac{q}{1+q}-\frac{1}{1+q} \cdot \frac{q_{1}}{r_{1}^{3}}-\frac{q}{1+q} \cdot \frac{q_{1}-1}{r_{2}^{3}} \\
\frac{d p_{2}}{d t} & =-p_{1}-\frac{1}{1+q} \cdot \frac{q_{2}}{r_{1}^{3}}-\frac{q}{1+q} \cdot \frac{q_{2}}{r_{2}^{3}} .
\end{aligned}
$$

The canonical equations have singularities in terms $\frac{1}{r_{1}}$ and $\frac{1}{r_{2}}$. These singularities can be eliminate by regularization.

\section{The regularization of the restricted three-body problem}

The procedure of regularization consists on two transformations: the coordinate transformation, which gives the shape of the orbit, and the time transformation, which 
makes the slow-down motion.

\subsection{The coordinate transformation}

The first step performed in the process of coordinate transformation consists in introduction of new coordinates $Q_{1}$ and $Q_{2}$. Let us introduce the generating function $\mathcal{S}$ (see Stiefel and Scheifele (1971), p.196):

$$
\mathcal{S}=-p_{1} f\left(Q_{1}, Q_{2}\right)-p_{2} g\left(Q_{1}, Q_{2}\right),
$$

a twice continuously differentiable function. Stieffel (15) write that, in order to obtain the regularized equations of motion it is necessary to impose only one condition on functions $f$ and $g$ : they must be harmonic and conjugated function, that means we have:

$$
\begin{aligned}
\frac{\partial f}{\partial Q_{1}} & =\frac{\partial g}{\partial Q_{2}} \\
\frac{\partial f}{\partial Q_{2}} & =-\frac{\partial g}{\partial Q_{1}} .
\end{aligned}
$$

The generating equations are

$$
q_{i}=-\frac{\partial \mathcal{S}}{\partial p_{i}}, \quad P_{i}=-\frac{\partial \mathcal{S}}{\partial Q_{i}}, \quad i \in\{1,2\},
$$

with $P_{1}, P_{2}$ as new generalized momenta, or explicitly

$$
\begin{aligned}
q_{1} & =-\frac{\partial \mathcal{S}}{\partial p_{1}}=f\left(Q_{1}, Q_{2}\right) \\
q_{2} & =-\frac{\partial \mathcal{S}}{\partial p_{2}}=g\left(Q_{1}, Q_{2}\right) \\
P_{1} & =-\frac{\partial \mathcal{S}}{\partial Q_{1}}=p_{1} \frac{\partial f}{\partial Q_{1}}+p_{2} \frac{\partial g}{\partial Q_{1}}=p_{1} a_{11}+p_{2} a_{12} \\
P_{2} & =-\frac{\partial \mathcal{S}}{\partial Q_{2}}=p_{1} \frac{\partial f}{\partial Q_{2}}+p_{2} \frac{\partial g}{\partial Q_{2}}=-p_{1} a_{12}+p_{2} a_{11}
\end{aligned}
$$

where

$$
a_{11}=\frac{\partial f}{\partial Q_{1}}=\frac{\partial g}{\partial Q_{2}}
$$




$$
a_{12}=-\frac{\partial f}{\partial Q_{2}}=\frac{\partial g}{\partial Q_{1}}
$$

Let introduce the following notation:

$$
\begin{gathered}
\mathbf{A}=\left(\begin{array}{cc}
a_{11} & a_{12} \\
-a_{12} & a_{11}
\end{array}\right), D=\operatorname{det} \mathbf{A}=a_{11}^{2}+a_{12}^{2} \\
\mathbf{p}=\left(\begin{array}{c}
p_{1} \\
p_{2}
\end{array}\right), \mathbf{P}=\left(\begin{array}{c}
P_{1} \\
P_{2}
\end{array}\right), \mathbf{p}=\frac{A^{T}}{D} \mathbf{P}, \mathbf{P}=A \cdot \mathbf{p}, p_{1}^{2}+p_{2}^{2}=\frac{P_{1}^{2}+P_{2}^{2}}{D}
\end{gathered}
$$

where $\mathbf{A}^{T}$ represents the transpose of matrix $\mathbf{A}$.

The new Hamiltonian with the generalized coordinates $Q_{1}$ and $Q_{2}$ and generalized momenta $P_{1}$ and $P_{2}$ is:

$$
\begin{aligned}
\mathcal{H}\left(Q_{1}, Q_{2}, P_{1}, P_{2}\right) & =\frac{1}{2 D}\left[P_{1}^{2}+P_{2}^{2}+P_{1} \frac{\partial}{\partial Q_{2}}\left(f^{2}+g^{2}\right)-P_{2} \frac{\partial}{\partial Q_{1}}\left(f^{2}+g^{2}\right)\right]+ \\
& +\frac{q}{1+q} f-\frac{1}{1+q} \cdot \frac{1}{\bar{r}_{1}}-\frac{q}{1+q} \cdot \frac{1}{\bar{r}_{2}}-\frac{q^{2}}{2(1+q)^{2}}
\end{aligned}
$$

where $\bar{r}_{1}=\sqrt{f^{2}+g^{2}}, \bar{r}_{2}=\sqrt{(f-1)^{2}+g^{2}}, D=a_{11}^{2}+a_{12}^{2}$ and the explicit canonical equations of motion in new variables become:

$$
\begin{aligned}
\frac{d Q_{1}}{d t} & =\frac{1}{2 D}\left[2 P_{1}+\frac{\partial}{\partial Q_{2}}\left(f^{2}+g^{2}\right)\right] \\
\frac{d Q_{2}}{d t} & =\frac{1}{2 D}\left[2 P_{2}-\frac{\partial}{\partial Q_{1}}\left(f^{2}+g^{2}\right)\right] \\
\frac{d P_{1}}{d t} & =-\frac{P_{1}}{2 D} \cdot \frac{\partial^{2}}{\partial Q_{1} \partial Q_{2}}\left(f^{2}+g^{2}\right)+\frac{P_{2}}{2 D} \cdot \frac{\partial^{2}}{\partial Q_{1} \partial Q_{1}}\left(f^{2}+g^{2}\right)-\frac{q}{1+q} \frac{\partial f}{\partial Q_{1}}+ \\
& +\frac{1}{1+q} \cdot \frac{\partial}{\partial Q_{1}}\left(\frac{1}{\bar{r}_{1}}\right)+\frac{q}{1+q} \cdot \frac{\partial}{\partial Q_{1}}\left(\frac{1}{\bar{r}_{2}}\right) \\
\frac{d P_{2}}{d t} & =-\frac{P_{1}}{2 D} \cdot \frac{\partial^{2}}{\partial Q_{2} \partial Q_{2}}\left(f^{2}+g^{2}\right)+\frac{P_{2}}{2 D} \cdot \frac{\partial^{2}}{\partial Q_{2} \partial Q_{1}}\left(f^{2}+g^{2}\right)-\frac{q}{1+q} \frac{\partial f}{\partial Q_{2}}+ \\
& +\frac{1}{1+q} \cdot \frac{\partial}{\partial Q_{2}}\left(\frac{1}{\bar{r}_{1}}\right)+\frac{q}{1+q} \cdot \frac{\partial}{\partial Q_{2}}\left(\frac{1}{\bar{r}_{2}}\right)
\end{aligned}
$$

So, in order to obtain the regularized equations of motion (18), it was necessary to impose only one condition on functions $f$ and $g$ : they must be harmonic conjugated functions. But there are a lot of harmonic conjugated functions. 
We can find harmonic and conjugate polynomial functions, by using the theory of complex functions. We denote $z=Q_{1}+i Q_{2}$ a single complex variable and $h: \Omega \rightarrow \mathbb{C}$, $h(z)=h\left(Q_{1}+i Q_{2}\right)=f\left(Q_{1}, Q_{2}\right)+i g\left(Q_{1}, Q_{2}\right)$ a complex-valued function. (Here $f$ and $g$ are two real functions, depending on two real variables $Q_{1}$ and $Q_{2}$.) From the theory of complex numbers (see Carathéodory (2001)) we know that, if $h(z)$ is a complex function, then its real and imaginary parts are harmonic functions. That means:

$$
\frac{\partial^{2} f\left(Q_{1}, Q_{2}\right)}{\partial Q_{1}^{2}}+\frac{\partial^{2} f\left(Q_{1}, Q_{2}\right)}{\partial Q_{2}^{2}}=0, \text { and } \frac{\partial^{2} g\left(Q_{1}, Q_{2}\right)}{\partial Q_{1}^{2}}+\frac{\partial^{2} g\left(Q_{1}, Q_{2}\right)}{\partial Q_{2}^{2}}=0
$$

Considering $h(z)=z$ it results $h\left(z^{n}\right)=z^{n}, n \in \mathbb{N}$ and $z^{n}=\left(Q_{1}+i Q_{2}\right)^{n}$. We obtain so the harmonic polynomials (see Table 1 , for $n=0,1, \ldots, 8$ ).

\section{Remarks:}

1. The case $n=0$ (in Table 1 ) isn't relevant for regularization.

2. If $n=1$ (in Table 1 ), the physical plane coincides with the regularized plane.

3. If $n=2$ (in Table 1 ), we obtain the Levi-Civita regularization (see (Levi-Civita 1906)).

4. It is easy to see that all pairs of functions $f\left(Q_{1}, Q_{2}\right)$ and $g\left(Q_{1}, Q_{2}\right)$ are conjugate, because they verify the relations of Cauchy-Riemann:

$$
\frac{\partial f}{\partial Q_{1}}=\frac{\partial g}{\partial Q_{2}} \text { and } \frac{\partial f}{\partial Q_{2}}=-\frac{\partial g}{\partial Q_{1}}
$$

In order to obtain the canonical equations, when $f$ and $g$ are harmonic and conjugate polynomial functions, we have to write first the corresponding Hamiltonian equation.

Let us consider the complex variable $z=Q_{1}+i Q_{2}$, which can be written in the trigonometric form: $z=\sqrt{Q_{1}^{2}+Q_{2}^{2}}(\cos (T)+i \sin (T))$, where $\tan (T)=\frac{Q_{2}}{Q_{1}}, i=\sqrt{-1}$. We 
denote $f_{n}=\Re\left(z^{n}\right)$ and $g_{n}=\Im\left(z^{n}\right), n \in \mathbb{N}, n \geq 2$. By consequence we obtain:

$$
f_{n}^{2}+g_{n}^{2}=\left(Q_{1}^{2}+Q_{2}^{2}\right)^{n}, \quad D_{n}=\left(\frac{\partial f_{n}}{\partial Q_{1}}\right)^{2}+\left(\frac{\partial f_{n}}{\partial Q_{2}}\right)^{2}=n^{2}\left(Q_{1}^{2}+Q_{2}^{2}\right)^{n-1} .
$$

The equation of Hamiltonian (eq. 17) becomes in this case:

$$
\begin{aligned}
\mathcal{H}\left(Q_{1}, Q_{2}, P_{1}, P_{2}\right) & =\frac{1}{2 D_{n}}\left(P_{1}^{2}+P_{2}^{2}\right)+\frac{P_{1} Q_{2}}{n}-\frac{P_{2} Q_{1}}{n}+\frac{q}{1+q} f_{n}- \\
& -\frac{1}{1+q} \cdot \frac{1}{\bar{r}_{1 n}}-\frac{q}{1+q} \cdot \frac{1}{\bar{r}_{2 n}}-\frac{q^{2}}{2(1+q)^{2}}
\end{aligned}
$$

and the canonical equations of motion will be:

$$
\begin{aligned}
\frac{d Q_{1}}{d t} & =\frac{P_{1}}{D_{n}}+\frac{Q_{2}}{n} \\
\frac{d Q_{2}}{d t} & =\frac{P_{2}}{D_{n}}-\frac{Q_{1}}{n} \\
\frac{d P_{1}}{d t} & =\frac{(n-1) Q_{1}\left(P_{1}^{2}+P_{2}^{2}\right)}{D_{n}\left(Q_{1}^{2}+Q_{2}^{2}\right)}+\frac{P_{2}}{n}-\frac{q}{1+q} \frac{\partial f_{n}}{\partial Q_{1}}+ \\
& +\frac{1}{(1+q)} \frac{\partial}{\partial Q_{1}}\left(\frac{1}{\bar{r}_{1 n}}\right)+\frac{q}{(1+q)} \frac{\partial}{\partial Q_{1}}\left(\frac{1}{\bar{r}_{2 n}}\right) \\
\frac{d P_{2}}{d t} & =\frac{(n-1) Q_{2}\left(P_{1}^{2}+P_{2}^{2}\right)}{D_{n}\left(Q_{1}^{2}+Q_{2}^{2}\right)}-\frac{P_{1}}{n}-\frac{q}{1+q} \frac{\partial f_{n}}{\partial Q_{2}}+ \\
& +\frac{1}{(1+q)} \frac{\partial}{\partial Q_{2}}\left(\frac{1}{\bar{r}_{1 n}}\right)+\frac{q}{(1+q)} \frac{\partial}{\partial Q_{2}}\left(\frac{1}{\bar{r}_{2 n}}\right)
\end{aligned}
$$

where $\bar{r}_{1 n}=\sqrt{f_{n}^{2}+g_{n}^{2}}, \quad \bar{r}_{2 n}=\sqrt{\left(f_{n}-1\right)^{2}+g_{n}^{2}}$.

As one can see these equations are still singular. In order to eliminate the singularities we must transform the time.

\subsection{The time transformation}

The second step performed in the process of regularization consists in the time transformation see (Stiefel and Scheifele 1971). In order to solve the canonical equations of 
motion, we introduce the fictitious time $\tau$, and make the time transformation $\frac{d t}{d \tau}=\bar{r}_{1 n}^{3} \bar{r}_{2 n}^{3}$. So, the regular equations of motion become:

$$
\begin{aligned}
\frac{d Q_{1}}{d \tau} & =\left(\frac{P_{1}}{D_{n}}+\frac{Q_{2}}{n}\right) \bar{r}_{1 n}^{3} \bar{r}_{2 n}^{3} \\
\frac{d Q_{2}}{d \tau} & =\left(\frac{P_{2}}{D_{n}}-\frac{Q_{1}}{n}\right) \bar{r}_{1 n}^{3} \bar{r}_{2 n}^{3} \\
\frac{d P_{1}}{d \tau} & =\left(\frac{(n-1) Q_{1}\left(P_{1}^{2}+P_{2}^{2}\right)}{D_{n}\left(Q_{1}^{2}+Q_{2}^{2}\right)}+\frac{P_{2}}{n}-\frac{q}{1+q} \frac{\partial f_{n}}{\partial Q_{1}}+\right. \\
& \left.+\frac{1}{(1+q)} \frac{\partial}{\partial Q_{1}}\left(\frac{1}{\bar{r}_{1 n}}\right)+\frac{q}{(1+q)} \frac{\partial}{\partial Q_{1}}\left(\frac{1}{\bar{r}_{2 n}}\right)\right) \bar{r}_{1 n}^{3} \bar{r}_{2 n}^{3} \\
\frac{d P_{2}}{d \tau} & =\left(\frac{(n-1) Q_{2}\left(P_{1}^{2}+P_{2}^{2}\right)}{D_{n}\left(Q_{1}^{2}+Q_{2}^{2}\right)}-\frac{P_{1}}{n}-\frac{q}{1+q} \frac{\partial f_{n}}{\partial Q_{2}}+\right. \\
& \left.+\frac{1}{(1+q)} \frac{\partial}{\partial Q_{2}}\left(\frac{1}{\bar{r}_{1 n}}\right)+\frac{q}{(1+q)} \frac{\partial}{\partial Q_{2}}\left(\frac{1}{\bar{r}_{2 n}}\right)\right) \bar{r}_{1 n}^{3} \bar{r}_{2 n}^{3}
\end{aligned}
$$

Now, the equations of motion of the test particle do not have singularities.

\section{Numerical application: trajectories of the test particle}

In order to obtain the trajectories in the regularized plane for different polynomial

functions $f$ and $g$, the canonical equations of motion of the test particle must be integrated, using initial conditions. We denote

$$
q_{10}=q_{1}(t) /_{t=0}, \quad q_{20}=q_{2}(t) /_{t=0}, \quad p_{10}=p_{1}(t) /_{t=0}, \quad p_{20}=p_{2}(t) /_{t=0}
$$

the initial conditions for the canonical equations (9), (10), (11), (12) in the physical plane, and

$$
Q_{10}=Q_{1}(t) /_{t=0}, \quad Q_{20}=Q_{2}(t) /_{t=0}, \quad P_{10}=P_{1}(t) /_{t=0}, \quad P_{20}=P_{2}(t) /_{t=0}
$$

the initial conditions for the canonical equations in the regularized plane. The connection between these initial conditions is given by the equations (see Roman et al. (2012)):

$$
q_{10}=f\left(Q_{10}, Q_{20}\right)
$$




$$
\begin{aligned}
q_{20} & =g\left(Q_{10}, Q_{20}\right) \\
P_{10} & =p_{10}\left(\frac{\partial f}{\partial Q_{1}}\right)_{\left(Q_{10}, Q_{20}\right)}+p_{20}\left(\frac{\partial g}{\partial Q_{1}}\right)_{\left(Q_{10}, Q_{20}\right)} \\
P_{20} & =-p_{10}\left(\frac{\partial g}{\partial Q_{1}}\right)_{\left(Q_{10}, Q_{20}\right)}+p_{20}\left(\frac{\partial f}{\partial Q_{1}}\right)_{\left(Q_{10}, Q_{20}\right)}
\end{aligned}
$$

Applying the transformation of coordinates given by $f$ and $g$, not only the trajectory will be changed, but the positions of the components of the binary system $S_{1}$ and $S_{2}$ will be changed too. In Table 2 there are given the positions of $S_{1}$ and $S_{2}$ in the regularized plane, for every type of transformation presented in Table 1 . Sometimes to one position of $S_{2}$ in the physical plane correspond two or more positions in the regularized plane. This fact is normal (see Stiefel and Scheifele (1971)), and is shown in Table 2.

Starting with the initial conditions in the physical plane:

$$
q_{10}=0.6, q_{20}=0.4, \quad p_{10}=0.1, \quad p_{20}=0.6
$$

we calculated the corresponding initial conditions in the regularized plane, for each regularization method presented in Table 1 . These new initial conditions are given in Table 2 , as well.

In Figure 1 there are presented the trajectories for each type of functions $f$ and $g$, given in Table 1. All the trajectories are represented for a time interval equal with an orbital period. For $n=1$ is represented the trajectory of the test particle in the physical plane. For $n=2$, the trajectory in the Levi-Civita regularized plane is given.

As we can see from Figure 1, by changing the degree $n$ of polynomial functions $f$ and $g$, the initial position $P_{0}$ is changed, but the shape of the trajectory maintains the same topology.

If $f$ and $g$ are polynomial functions of $n$ degree, the greater is $n$, the greater is the distance of $P_{0}$ to the origin of the coordinate system. 

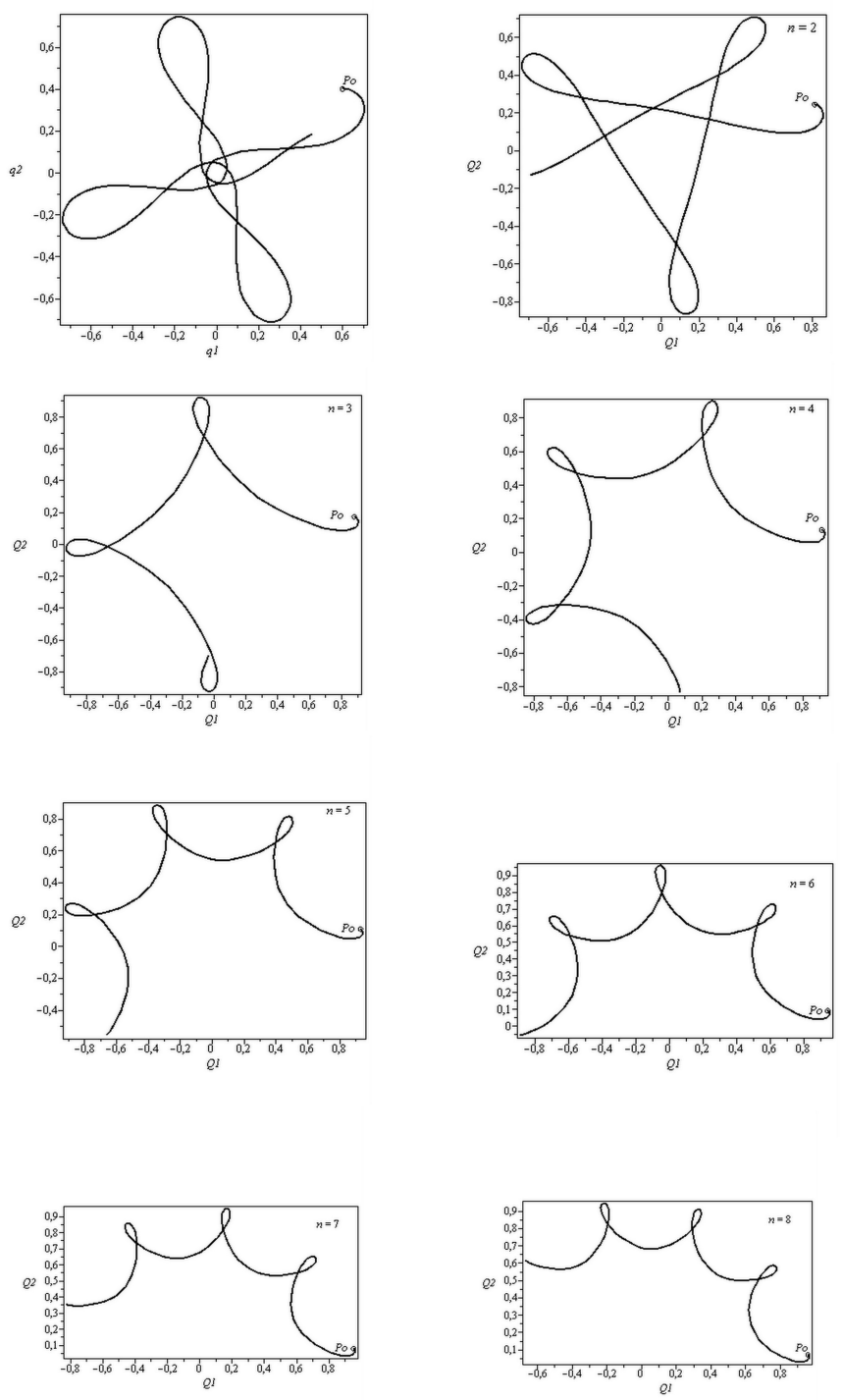

Fig. 1.- Trajectories of the test particle, when $f$ and $g$ are polynomial functions of order $n=2 \ldots 8$ 
In Roman et al. (2012) there are presented some considerations on the geometrical transformation for Levi-Civita regularization. In the following we generalize two results concerning the polar radii and the polar angles for each values of polynomial degrees (see Figure 2).
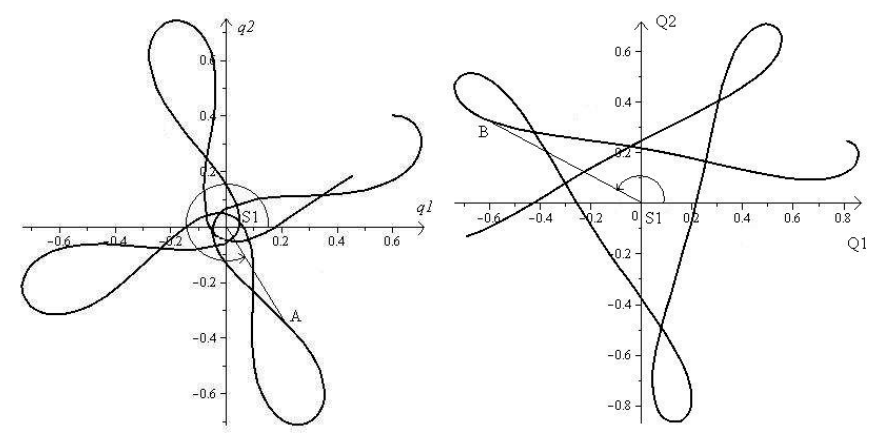

Fig. 2.- How to obtain the B point in (Q1, S1, Q2) plane from the A point in (q1, S1, q2) plane, for $n=2$

\section{Theorem}

In the polynomial regularization's methods, if $A$ is an arbitrary point of the trajectory in the physical plane $(n=1)$, and $B$ is its corresponding point in the regularized plane (see Figure 2), then we have the following relations concerning the polar radii and angles:

$$
\left|S_{1} A\right|=\left|S_{1} B\right|^{n}, \quad \widehat{A S_{1} q_{1}}=n \cdot \widehat{B S_{1} Q_{1}}, \quad \forall n \in \mathbb{N}, \quad n \geq 1 .
$$

Proof:

We have:

$$
\begin{gathered}
\left|S_{1} A\right|=\sqrt{q_{1}^{2}+q_{2}^{2}}, \quad\left|S_{1} B\right|=\sqrt{Q_{1}^{2}+Q_{2}^{2}}, \\
q_{1}=f_{n}=\left(\sqrt{Q_{1}^{2}+Q_{2}^{2}}\right)^{n} \cos (n T), \quad q_{2}=g_{n}=\left(\sqrt{Q_{1}^{2}+Q_{2}^{2}}\right)^{n} \sin (n T)
\end{gathered}
$$

Then

$$
\left|S_{1} A\right|=\sqrt{q_{1}^{2}+q_{2}^{2}}=\left(\sqrt{Q_{1}^{2}+Q_{2}^{2}}\right)^{n}\left(\sin ^{2}(n T)+\cos ^{2}(n T)\right)=
$$




$$
=\left(\sqrt{Q_{1}^{2}+Q_{2}^{2}}\right)^{n}=\left|S_{1} B\right|^{n}
$$

On the other hand

$$
\begin{aligned}
\tan \left(\widehat{A S_{1} q_{1}}\right) & =\frac{q_{2}}{q_{1}}=\frac{g_{n}}{f_{n}}=\tan (n T)=\tan \left(n \arctan \left(\frac{Q_{2}}{Q_{1}}\right)\right)= \\
& =\tan \left(n \widehat{B S_{1} Q_{1}}\right),
\end{aligned}
$$

and then: $\widehat{A S_{1} q_{1}}=n \cdot \widehat{B S_{1} Q_{1}}+k \pi, k \in \mathbb{Z}$, or

$$
\widehat{B S_{1} Q_{1}}=\frac{1}{n} \widehat{A S_{1} q_{1}}-\frac{k \pi}{n}, \quad k \in \mathbb{Z}
$$

From the notation $f_{n}=\Re\left(z^{n}\right)$ and $g_{n}=\Im\left(z^{n}\right), n \in \mathbb{N}, n \geq 2$ we can see that to one point in the regularized plane (one couple $\left(Q_{1}, Q_{2}\right)$ ) it corresponds only one point in the physical plane (one couple $\left(q_{1}, q_{2}\right)$ ), then in equation $23 k$ must have only one value, for all values of $n$. If $n=1$ the physical plane is transformed in physical plane (the identical transformation), and for this transformation $\widehat{B S_{1} Q_{1}}=\widehat{A S_{1} q_{1}}$. It results that the unique value of $k$ must be $k=0$. Then, $\widehat{B S_{1} Q_{1}}=\widehat{A S_{1} q_{1}}, \forall n \in \mathbb{N}, \quad n \geq 1$.

$$
\text { If } n=2 \text { one can obtain the Levi-Civita's result. }
$$

\section{Conclusion}

Because the unique condition imposed to the generating functions $f$ and $g$ is to be harmonic and conjugate, it exists a whole family of polynomial functions which can be selected. These polynomial functions of $n$ degree generate trajectories having the same topology. The greater is the degree of the polynomial, the greater is the distance of initial start point to the origin of the coordinate system.

The polar radius in the physical plane is equal whith the polar radius in the regularized plane, raised to the $n$ power. 
The polar angle in the physical plane is $n$ times greater than the polar angle in the regularized plane.

For $n=2$ the Levi-Civita regularization methods is found.

Even if Levi-Civita regularization method is more easy to use, from theoretical point of view it is interesting to encapsulate this method in a family of methods which all conserve the Levi-Civita method's properties.

The authors wish to acknowledge the anonymous reviewer for his/her helpful comment to the manuscript. 
Table 1: Some harmonic and conjugate polynomial functions

\begin{tabular}{lll}
$n$ & $f\left(Q_{1}, Q_{2}\right)$ & $g\left(Q_{1}, Q_{2}\right)$ \\
\hline$n=0$ & 1 & 0 \\
$n=1$ & $Q_{1}$ & $Q_{2}$ \\
$n=2$ & $Q_{1}^{2}-Q_{2}^{2}$ & $2 Q_{1} Q_{2}$ \\
$n=3$ & $Q_{1}^{3}-3 Q_{1} Q_{2}^{2}$ & $3 Q_{1}^{2} Q_{2}-Q_{2}^{3}$ \\
$n=4$ & $Q_{1}^{4}-6 Q_{1}^{2} Q_{2}^{2}+Q_{2}^{4}$ & $4 Q_{1}^{3} Q_{2}-4 Q_{1} Q_{2}^{3}$ \\
$n=5$ & $Q_{1}^{5}-10 Q_{1}^{3} Q_{2}^{2}+5 Q_{1} Q_{2}^{4}$ & $5 Q_{1}^{4} Q_{2}-10 Q_{1}^{2} Q_{2}^{3}+Q_{2}^{5}$ \\
$n=6$ & $Q_{1}^{6}-15 Q_{1}^{4} Q_{2}^{2}+15 Q_{1}^{2} Q_{2}^{4}-Q_{2}^{6}$ & $6 Q_{1}^{5} Q_{2}-20 Q_{1}^{3} Q_{2}^{3}+6 Q_{1} Q_{2}^{5}$ \\
$n=7$ & $Q_{1}^{7}-21 Q_{1}^{5} Q_{2}^{2}+35 Q_{1}^{3} Q_{2}^{4}-7 Q_{1} Q_{2}^{6}$ & $7 Q_{1}^{6} Q_{2}-35 Q_{1}^{4} Q_{2}^{3}+21 Q_{1}^{2} Q_{2}^{5}-Q_{2}^{7}$ \\
$n=8$ & $Q 1^{8}-28 Q 1^{6} Q 2^{2}+70 Q 1^{4} Q 2^{4}-$ & $8 Q 1^{7} Q 2-56 Q 1^{5} Q 2^{3}+56 Q 1^{3} Q 2^{5}-$ \\
& $-28 Q 1^{2} Q 2^{6}+Q 2^{8}$ & $-8 Q 1 Q 2^{7}$ \\
\hline
\end{tabular}


Table 2: $S_{1}, S_{2}$ positions and the initial conditions for different methods of regularization (the case $n=1$ corresponds to the physical plane)

\begin{tabular}{llcc}
\hline Type & $S_{1}$ & $S_{2}[a$ & $\left(Q_{10}, Q_{20}, P_{10}, P_{20}\right]^{b}$ \\
\hline Pol. function, $n=1$ & $(0,0)$ & $(1,0)$ & $(0.6,0.4,0.1,0.6)$ \\
Pol. function, $n=2$ & $(0,0)$ & $S_{2}^{k}, k \in\{1,2\}$ & $(0.812,0.246,0.457,0.926)$ \\
Pol. function, $n=3$ & $(0,0)$ & $S_{2}^{k}, k \in\{1, \ldots, 3\}$ & $(0.879,0.174,0.775,1.245)$ \\
Pol. function, $n=4$ & $(0,0)$ & $S_{2}^{k}, k \in\{1, \ldots, 4\}$ & $(0.911,0.135,1.084,1.564)$ \\
Pol. function, $n=5$ & $(0,0)$ & $S_{2}^{k}, k \in\{1, \ldots, 5\}$ & $(0.930,0.109,1.389,1.884)$ \\
Pol. function, $n=6$ & $(0,0)$ & $S_{2}^{k}, k \in\{1, \ldots, 6\}$ & $(0.942,0.092,1.693,2.203)$ \\
Pol. function, $n=7$ & $(0,0)$ & $S_{2}^{k}, k \in\{1, \ldots, 7\}$ & $(0.950,0.080,1.995,2.523)$ \\
Pol. function, $n=8$ & $(0,0)$ & $S_{2}^{k}, k \in\{1, \ldots, 8\}$ & $(0.957,0.070,2.297,2.843)$ \\
\hline
\end{tabular}

${ }^{a}$ where $S_{2}^{k}\left(\cos \frac{2 \pi(k-1)}{n}, \sin \frac{2 \pi(k-1)}{n}\right), \quad k \in\{1, \ldots, n\}$

${ }^{b}$ calculated only for $k=1$ 


\section{REFERENCES}

Arenstorf, R. F.: AJ 68, 548 (1963)

Birkhoff, G. D.: Rend. Circ. Mat. Palermo 39, 1 (1915)

Boccaletti, D., Pucacco, G.: Theory of orbits 1. Springer-Verlag Berlin Heidelberg New York (1996)

Burrau, C.: Vierteljahrschrift Astron. Ges. 41, 261 (1906)

Carathéodory: Theory of functions of a complex variable, Vol. 1, 304 pages, AMS Chelsea Publishing, Providence, Rhode Island (2001)

Csillik, I.: Regularization methods in celestial mechanics, Cluj: House of the Book of Science (2003)

Érdi, B.: Celestial Mechanics 90, 35 (2004)

Kustaanheimo, P., Stiefel, E. L.: J. Reine Angewandte Math. 218, 204 (1965)

Lemaître, G.: Vistas in Astronomy 1, 207 (1955)

Levi-Civita, T.: Acta Mathematica 30, 305 (1906)

Mioc, V., Csillik, I.: RoAJ 12, 167 (2002)

Roman, R., Ap\&SS 335, 475 (2011)

Roman, R., Szücs-Csillik, I.: Ap\&SS 338, 233 (2012)

Szücs-Csillik, I., Roman, R.: RoAJ 22, 2, 145 (2012)

Stiefel, L., Scheifele, G.: Linear and regular celestial mechanics. Springer Berlin (1971)

Szebehely, V.: Theory of orbits. Academic Press, New York (1967) 
Thiele, T. N.: Astron. Nachr. 138, 1 (1896) 